\title{
Biofumigación con Brassica juncea: efecto sobre la flora arvense
}

\author{
Perniola, Omar Salvador ${ }^{1,4}$; Silvia Elena Chorzempa ${ }^{2}$; Sebastián Staltari ${ }^{1}$; María del \\ Carmen Molina ${ }^{1,3}$
}

${ }^{1}$ Instituto Fitotécnico de Santa Catalina, Facultad de Ciencias Agrarias y Forestales, UNLP. Garibaldi 3400, Llavallol, CP 1836, Buenos Aires, Argentina; ${ }^{2}$ Facultad de Ciencias Agrarias, UNLZ. Ruta № 4, Km 2 , Llavallol, CP 1836, Buenos Aires, Argentina; ${ }^{3}$ CONICET; ${ }^{4}$ omarperniola@yahoo.com.ar

Perniola, Omar Salvador; Silvia Elena Chorzempa; Sebastián Staltari; María del Carmen Molina (2019) Biofumigación con Brassica juncea: efecto sobre la flora arvense. Rev. Fac. Agron. Vol 118 (1): 25-35.

\begin{abstract}
La biofumigación con brassicáceas es una táctica biológica que podría contribuir al manejo integrado de malezas en las etapas iniciales de los cultivos hortícolas. El objetivo de este trabajo fue determinar la dosis del biofumigante Brassica juncea L. Czerniak (mostaza parda) técnicamente más eficaz para el manejo de malezas en un sustrato. El ensayo se realizó en cajones de madera que contenían tierra extraída de la capa arable de un suelo de Llavallol, Argentina, que portaba las semillas de la flora del lugar. Se trituraron plantas de mostaza parda y se mezclaron con la tierra, en dosis de 2,5, 5, 10 y $15 \mathrm{~kg} \cdot \mathrm{m}^{-2}$. Cada cajón se regó y cubrió con polietileno negro durante 21 días. Paralelamente, se evaluó un control sin biofumigante, con idéntica cobertura y riego. Tres semanas después de extraer el polietileno se contabilizó la cantidad y frecuencia de individuos de cada especie arvense y se midió cobertura y peso seco por tratamiento. La dosis de $2,5 \mathrm{~kg} \cdot \mathrm{m}^{-2}$ redujo el número total de individuos de arvenses mono y dicotiledóneas, en particular de Digitaria sanguinalis (L.) Scop. (pasto de cuaresma), Portulaca oleracea L. (verdolaga) y Taraxacum officinalis G.H. Weber ex Wiggers (diente de león), aunque no disminuyó significativamente la biomasa aérea y la cobertura. El tratamiento con $5 \mathrm{~kg} \cdot \mathrm{m}^{-2}$ mostró un comportamiento similar al control. Las dosis de 10 y $15 \mathrm{~kg} \cdot \mathrm{m}^{-2}$ favorecieron el crecimiento de las malezas. La biofumigación con $2,5 \mathrm{~kg} \cdot \mathrm{m}^{-2}$ representa una herramienta alternativa para el manejo integrado de malezas en estadios iniciales de cultivos hortícolas.
\end{abstract}

Palabras clave: malezas, control biológico, brassicáceas, mostaza parda, manejo integrado de malezas.

Perniola, Omar Salvador; Silvia Elena Chorzempa; Sebastián Staltari; María del Carmen Molina (2019) Biofumigation with Brassica juncea: effect on weeds. Rev. Fac. Agron. Vol 118 (1): 25-35.

\begin{abstract}
Biofumigation with Brassicaceae is a biological tactic which could contribute to the integrated weeds management in the initial stages of horticultural crops. The aim of this study was to determine the dose of the biofumigant Brassica juncea L. Czerniak (brown mustard) technically more effective for the weeds management in a substrate. The test was carried out in wooden drawers that containing ground extracted from the arable layer of a soil of Llavallol, Argentina and including the seeds of the local flora. Brown mustard plants were triturated and mixed with the ground in doses of 2.5, 5,10 and $15 \mathrm{~kg} \cdot \mathrm{m}^{-2}$. Each drawer was irrigated and covered with black polyethylene for 21 days. At the same time, a control without biofumigant, with identical coverage and irrigation was evaluated. Three weeks after extracting polyethylene, the amount and frequency of individuals of each species of weed were counted and the cover and dry weight by treatment were measured. The biofumigant treatment with $2.5 \mathrm{~kg} \cdot \mathrm{m}^{-2}$ reduced the total number of individuals of mono and dicotyledonous weeds, in particular of Digitaria sanguinalis (L.) Scop. (large crabgrass), Portulaca oleracea L. (common purslane) y Taraxacum officinalis G.H. Weber ex Wiggers (dandelion), although it did not generate significant reduction of aerial biomass and coverage. The treatment with $5 \mathrm{~kg} \cdot \mathrm{m}^{-2}$ showed an action similar to the control. Doses of 10 and $15 \mathrm{~kg} \cdot \mathrm{m}^{-2}$ favored the growth of weeds. Biofumigation with $2.5 \mathrm{~kg} \cdot \mathrm{m}^{-2}$ represents an alternative tactic for the integrated weeds management in the initial stages of horticultural crops.
\end{abstract}

Keywords: weeds, biological control, Brassicaceae, brown mustard, integrated weed management.

https://doi.org/10.24215/16699513e003

https://revistas.unlp.edu.ar/revagro

Recibido: 30/11/2017

Aceptado: $19 / 10 / 2018$

Disponible on line: $01 / 07 / 2019$

ISSN 0041-8676 - ISSN (on line) 1669-9513, Facultad de Ciencias Agrarias y Forestales, UNLP, Argentina 


\section{INTRODUCCIÓN}

Las malezas, o flora arvense, representan una de las principales adversidades de la producción hortícola. Compiten con las especies de cultivo por los recursos, pueden producir sustancias alelopáticas nocivas y hospedar enfermedades e insectos plaga, lo que conlleva a mermas en los rendimientos y en la calidad de la producción (Menalled, 2010). Pero las malezas también cumplen funciones ecológicas específicas en el agroecosistema hortícola: evitan la erosión del suelo, son hospedantes de insectos benéficos, constituyen un banco de diversidad genética, modifican el microclima local y regulan procesos hidrológicos (Altieri \& Nicholls, 1999; Labrador Moreno \& Altieri, 1994). Para minimizar las pérdidas económicas ocasionadas por las arvenses, sin anular las importantes funciones ecológicas de estas plantas, es necesario establecer normas de convivencia interespecífica cultivo - maleza, que implican reducir la germinación y el crecimiento de las malezas durante los períodos críticos de competencia (Blanco \& Leyva, 2007). Para la mayoría de las especies de hortalizas, el período crítico de competencia de malezas es equivalente al primer tercio de su ciclo vegetativo, aunque su duración es variable y depende de la especie y cultivar, tasa de crecimiento, composición de la flora arvense, etc. (Labrada, 1996). Por lo tanto, el manejo de malezas en las primeras etapas de un cultivo hortícola es fundamental para lograr un buen establecimiento y evitar mermas en el rendimiento.

Durante muchos años, la fumigación de suelos y sustratos con bromuro de metilo fue una práctica generalizada y de buenos resultados, para controlar malezas y otras plagas en las primeras etapas de los cultivos hortícolas, especialmente en producciones bajo invernáculo. Debido a la prohibición del uso del bromuro de metilo, a causa de su efecto nocivo sobre la capa de ozono (MBTOC, 1994), se lo sustituyó gradualmente por algunos pesticidas sintéticos $y$, en menor medida, por otros métodos, como la solarización y la inyección de vapor de agua (Biaggi et al., 2011; Pizano, 2014). Es importante continuar con la búsqueda de nuevas alternativas para el manejo integrado de malezas, que sean eficaces y amigables con el medio ambiente.

Una de esas alternativas es la biofumigación con brassicáceas, que puede definirse como el control de plagas y patógenos edáficos por medio de la incorporación en el suelo de ciertas especies de brassicáceas, que liberan diferentes tipos de isotiocianatos con actividad biocida, como resultado de la hidrólisis de los glucosinolatos contenidos en sus tejidos (Kirkegaard et al., 1993). A diferencia de los fumigantes de suelo sintéticos, como el metam sodio y el dazomet, la incorporación de enmiendas biofumigantes mejora la estructura y la penetración del agua, aumenta el contenido de materia orgánica del suelo y reduce el encostramiento (Pung et al., 2008). Durante el proceso de biofumigación, los glucosinolatos presentes en los tejidos de las brassicáceas (Kjaer, 1976), se hidrolizan por la acción de la enzima mirosinasa produciendo diferentes tipos de isotiocianatos, con variable grado de toxicidad frente a diversos organismos (Molina-Vargas \& BenturaCastellanos, 2009).

Según Gimsing \& Kirkegaard (2009), la efectividad de la biofumigación se basa en lograr la máxima hidrólisis de los glucosinolatos presentes en el tejido vegetal, para generar altas concentraciones de isotiocianatos en el suelo después de su incorporación; esto es favorecido por una gran disrupción celular, la adición de agua y alta temperatura del suelo. Price et al. (2005) hallaron que la concentración de allyl isotiocianato (AITC), generado a partir del tejido de Brassica juncea $\mathrm{L}$. Czerniak (mostaza parda), en un suelo a $45^{\circ} \mathrm{C}$ fue $81 \%$ superior que a $15^{\circ} \mathrm{C}$. La efectividad de la biofumigación también está condicionada por la duración del efecto biocida de los isotiocianatos en el suelo. Al respecto, los isotiocianatos son fuertemente adsorbidos por las arcillas y son propensos a pérdidas por volatilización y degradación microbiana (Gimsing \& Kirkegaard, 2009). Por lo tanto, la textura y microbiología del suelo influyen sobre la efectividad de la biofumigación, como así también la utilización de coberturas de suelo que impidan las pérdidas por volatilización. Price et al. (2005) observaron que la concentración de AITC fue un $38 \%$ mayor en un suelo franco arenoso comparado con un suelo franco arcilloso, $56 \%$ más alta en los tratamientos con cobertura plástica que en los que no presentaban cobertura y tres veces mayor en suelos autoclavados que en no autoclavados.

La biofumigación puede inhibir la emergencia y crecimiento de malezas (Anderson et al., 2008; Boydston et al., 2008; 2011; Brown \& Morra, 1995; Norsworthy et al., 2005; Perniola et al., 2011; 2016; Webber et al., 2012). También se ha demostrado la eficacia de esta técnica para reducir las poblaciones de nematodos (Mitidieri et al., 2009; Pattison et al., 2003) y hongos fitopatógenos (Charron \& Sams, 1999; Iriarte et al., 2011; Kirkegaard et al., 1996; Lafi et al., 2017; Mayton et al., 1996; Perniola et al., 2012; 2014).

Entre las especies de brassicáceas más estudiadas como biofumigantes se encuentran $B$. juncea y Sinapis alba L. (mostaza blanca). Según Björkman et al. (2015), los cultivos de cobertura de mostaza parda o blanca tienen un valor potencial para suprimir el crecimiento de malezas, pero la capacidad de supresión varía con la ubicación (localidad), el año y la fecha de siembra de la mostaza. Handiseni et al. (2011) observaron que la harina de semilla de mostaza (HSM) de B. juncea tuvo significativamente mayor eficacia como herbicida de poáceas que la HSM de $S$. alba, que fue más efectiva en el control de malezas de hoja ancha.

Numerosos investigadores han comprobado el efecto supresor de la biofumigación con mostazas sobre la germinación y el crecimiento de las arvenses. Anderson et al. (2008) observaron reducción de la población de Digitaria sanguinalis (L.) Scop. cuando incorporaron plantas maceradas de $B$. juncea en un suelo arenoso. Boydston et al. (2008) estudiaron el efecto de la harina de semilla de mostaza (HSM) de S. alba "IdaGold" sobre la emergencia de algunas malezas y observaron reducción del número de plántulas emergidas de $P o a$ annua L. y Stellaria media L. Earlywine et al. (2010) determinaron que la biofumigación con HSM de $B$. juncea suprimiría la emergencia y el crecimiento de varias especies de malezas comunes en el césped y tendría potencial selectividad para Cynodon dactylon 
(L.) Pers. En pruebas en invernadero con HSM de $S$. alba, Yu \& Morishita (2014) hallaron menores tasas de emergencia de Kochia scoparia (L.) Schrad. Chenopodium album L. y Echinochloa crus-galli (L.) Beauv., en comparación con el testigo sin mostaza. Boydston et al. (2011) aplicaron HSM de S. alba en plantaciones de cebolla y observaron reducción de la cantidad de malezas emergidas, pero también lesiones y mermas en el rendimiento del cultivo, variables según el estadio fenológico de la cebolla y el año ensayado. En un ensayo in vitro, Lefebvre et al. (2013) biofumigaron semillas de cinco especies (C. album, Ambrosia artemisiifolia L., Setaria viridis [L.] P. Beauv., Vicia cracca L. y Daucus carota L.) con plantas secas trituradas de $B$. juncea en floración, y observaron reducción de la germinación y de la supervivencia de semillas dormidas e incremento de la mortalidad de plántulas.

Si bien la mayoría de los estudios realizados en el tema, demuestran una acción eficaz de la biofumigación sobre el control de malezas, otros investigadores han hallado escaso efecto herbicida de esta táctica de biocontrol. Hoagland et al. (2008) hallaron que la HSM de $B$. juncea no suprimió la emergencia de malezas. Haramoto \& Gallandt (2005) observaron que los residuos de brassicáceas retrasan la emergencia y reducen el establecimiento de las malezas, aunque la magnitud de sus efectos es comparable a la de otros cultivos de cobertura, como Trifolium incarnatum L., Avena sativa L. y Fagopyrum esculentum Moench. Geary et al. (2008) observaron que la incorporación de un cultivo de mostaza blanca "IdaGold" previo a la plantación de cebolla produjo un escaso control de malezas. Según Bangarwa \& Norsworthy (2014) la biofumigación con brassicáceas proporciona un control marginal de las malezas por lo que debería complementarse con otras estrategias, como herbicidas, solarización, labores mecánicas, etc.

Actualmente, la información disponible sobre el manejo de malezas mediante biofumigación es insuficiente y de resultados ambiguos, por lo que resulta importante investigar en esta área, dado las ventajas ecológicas que presenta la biofumigación sobre los productos químicos sintéticos.

Debido a que son numerosas las variables que influyen sobre el proceso de biofumigación, es necesario establecer metodologías de manejo, adaptables a las condiciones locales, que contemplen el tipo de material (planta fresca, seca, subproducto industrial), especie botánica, estadio fenológico y dosis del biofumigante a utilizar, el procedimiento apropiado para el triturado e incorporación, el tiempo de cobertura con polietileno, etc. En ensayos realizados por nuestro grupo de trabajo en la zona de Llavallol, Argentina (Perniola et al., 2011; 2012; 2014; 2016; inédito, 2018) se observó que el cultivo de $B$. juncea registró menor incidencia y severidad de podredumbre negra (Xanthomonas campestris pv. campestris [Pammel] Dowson [Xcc]) y produjo mayor rendimiento de biomasa aérea fresca $\left(1,7\right.$ a $\left.2,3 \mathrm{~kg} \cdot \mathrm{m}^{-2}\right)$ que el cultivo de $S$. alba. En un ensayo in vitro (Perniola et al., 2016) se concluyó que la biofumigación con biomasa aérea fresca de $B$. juncea, en el estadio de fin de fructificación, presentó un notable efecto supresor sobre la germinación y el crecimiento de algunas malezas, superior al de $S$. alba.
El objetivo de este trabajo fue determinar el efecto biofumigante de diferentes dosis de plantas frescas de $B$. juncea, en el estadio de fin de fructificación, sobre la densidad, biomasa y cobertura de la flora arvense de un sustrato, a fin de hallar la dosis técnicamente más eficaz para el manejo de malezas.

\section{METODOLOGÍA}

Se llevó a cabo un ensayo en un invernáculo vidriado ubicado en el campo experimental del Instituto Fitotécnico de Santa Catalina (IFSC), Llavallol,

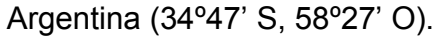

Las unidades experimentales fueron cajones de madera de $60 \mathrm{~cm}$ de largo, $40 \mathrm{~cm}$ de ancho y $13 \mathrm{~cm}$ de profundidad, rellenados con un sustrato que contenía semillas de la flora arvense del lugar y distintas cantidades de plantas trituradas de $B$. juncea.

Para la obtención del sustrato se roturó con un motocultivador, la capa arable del suelo $(10 \mathrm{~cm}$ superiores) de un sector del campo experimental y posteriormente se tamizó la tierra con una zaranda de 1 x $1 \mathrm{~cm}$. Se oreó al sol sobre un polietileno negro durante tres días, hasta alcanzar una humedad del $13,5 \%$ y se dispuso en los cajones.

El material vegetal utilizado como biofumigante se obtuvo de un cultivo de $B$. juncea sembrado en el IFSC en junio de 2016. Cuando el cultivo alcanzó el estadio de fin de fructificación (octubre de 2016) se segó con una hoz la parte aérea, se dispuso en andanas e inmediatamente se trituró con una cortadora de césped. En cada cajón se colocaron 24 litros de sustrato (tierra tamizada y oreada) y la correspondiente dosis del material fresco triturado de $B$. juncea. Se evaluaron cuatro dosis del biofumigante: $2,5,5,10$ y $15 \mathrm{~kg} \cdot \mathrm{m}^{-2}$ de biomasa fresca, equivalente a 475, 950, 1900 y 2850 g. $\mathrm{m}^{-2}$ de biomasa seca ó $2,75,5,5,11$ y $16,5 \%$ (peso fresco del biofumigante/peso del sustrato) ó $0,52,1,05$, 2,09 y $3,14 \%$ (peso seco del biofumigante/peso del sustrato), respectivamente. Posteriormente cada cajón se regó con 2,4 litros de agua y se cubrió con polietileno negro durante 21 días ("etapa biofumigante"). Para el tratamiento control se siguió la misma técnica pero sin el agregado de $B$. juncea.

Finalizada la etapa biofumigante, se extrajeron los polietilenos $\mathrm{y}$, durante las siguientes tres semanas, se regaron los cajones para mantener el sustrato cercano a capacidad de campo ("etapa post-biofumigante"). Al $21{ }^{\text {er }}$ día de la etapa post-biofumigante, se contabilizó la cantidad y frecuencia de individuos de cada especie arvense y se midió la cobertura total. Luego, se segó la biomasa aérea del total de malezas y se secó en estufa a $60^{\circ} \mathrm{C}$, hasta alcanzar un peso constante (peso seco). Todos los resultados se expresaron por unidad de superficie $\left(\mathrm{m}^{2}\right)$.

Para la medición de la cobertura se utilizó el programa CobCal versión 2.1 (Ferrari et al., 2009).

Con el objetivo de determinar la composición potencial de especies arvenses presentes en el sustrato e identificar las malezas de mayor importancia, se rellenaron tres cajones con 24 I del mismo sustrato utilizado en el ensayo y se regaron durante tres semanas para mantener el sustrato cercano a capacidad de campo. Al $21 .^{\text {er }}$ día se contabilizó la 
cantidad y frecuencia de individuos de cada especie arvense y se calculó el índice de valor de importancia (IVI) (Anzalone et al., 2011; Páez, 2001). El IVI es un formato numérico que permite asignarle a cada especie su categoría de importancia en base a su relación con las otras especies presentes en un área determinada (Anzalone et al., 2011). EI IVI se calculó como la suma de la densidad relativa y la frecuencia relativa de cada especie (Mostacedo \& Fredericksen, 2000), donde:

- Densidad relativa $=$ (densidad por especie $/$ densidad total) * 100

- Densidad por especie $=n^{\circ}$ de plantas por especie $/$ unidad de área $\left(\mathrm{m}^{2}\right)$

- $\quad$ Densidad total $=\sum$ densidad por especie

- Frecuencia relativa $=$ (frecuencia por especie $/$ frecuencia total) ${ }^{*} 100$

- Frecuencia por especie $=n^{\circ}$ de repeticiones en las que aparece la especie * $100 / \mathrm{n}^{\circ}$ total de repeticiones

- Frecuencia total $=\sum$ frecuencia por especie

Las especies que registraron un IVI mayor a 10 se evaluaron individualmente mediante la comparación de la densidad por especie entre tratamientos.

Durante el desarrollo del ensayo se midieron las temperaturas diarias máximas y mínimas dentro del invernáculo.

Se utilizó un diseño experimental completamente aleatorizado, que incluyó los 5 tratamientos, cada uno con tres repeticiones. Se practicó ANOVA simple y las medias se compararon mediante la prueba de Tukey. Cuando los datos no cumplieron con los supuestos de aleatoriedad, homocedasticidad y normalidad, se transformaron mediante la aplicación de logaritmo o arcoseno de raíz cuadrada y se sometieron al mismo análisis estadístico. Se efectuaron contrastes ortogonales para comparar pares de tratamientos en particular. Los datos que tras haber sido transformados no cumplieron con los supuestos, fueron analizados con la prueba no paramétrica de Kruskal-Wallis. En todos los casos, se utilizó el programa Statistica 7.

\section{RESULTADOS}

Durante la etapa biofumigante (24 de octubre al 14 de noviembre) la temperatura máxima media fue $32^{\circ} \mathrm{C}$ y la mínima media $14,9^{\circ} \mathrm{C}$. En la etapa post-biofumigante (14 de noviembre al 5 de diciembre) la temperatura máxima media fue $35,1^{\circ} \mathrm{C}$ y la mínima media $18^{\circ} \mathrm{C}$.

\section{Cantidad total de individuos de arvenses}

La biofumigación con $2,5 \mathrm{~kg} \cdot \mathrm{m}^{-2}$ registró la menor cantidad de individuos de arvenses $(61,36 \%$ menos que el control) y se diferenció significativamente del control $y$ de los demás tratamientos $\left(p \leq 8,37 \times 10^{-4}\right)$. Las dosis más altas de $B$. juncea (10 y $15 \mathrm{~kg} . \mathrm{m}^{-2}$ ) registraron las mayores cantidades de individuos de arvenses y se diferenciaron significativamente del control y de los otros tratamientos $\left(p \leq 5,08 \times 10^{-3}\right)$. La biofumigación con $5 \mathrm{~kg} \cdot \mathrm{m}^{-2}$ no se diferenció significativamente del control (Figura 1-A).
Cantidad de individuos de arvenses edóneas

En la flora arvense del sustrato evaluado, las especies monocotiledóneas representaron el $47,12 \%$ del IVI total (Tabla 1).

La biofumigación con $2,5 \mathrm{~kg} \cdot \mathrm{m}^{-2}$ registró la menor cantidad de individuos de arvenses monocotiledóneas $(80,55 \%$ menos que el control) y se diferenció significativamente del control y de los tratamientos con 10 y $15 \mathrm{~kg} \cdot \mathrm{m}^{-2}$ ( $\left.\mathrm{p} \leq 0,026\right)$, pero no de la biofumigación con $5 \mathrm{~kg} \cdot \mathrm{m}^{-2}$. Las dosis de 5,10 y $15 \mathrm{~kg} \cdot \mathrm{m}^{-2}$ no se diferenciaron significativamente del control (Figura 1-B).

\section{Cantidad de individuos de arvenses dicotiledóneas}

En la flora arvense del sustrato evaluado, las especies dicotiledóneas representaron el $52,88 \%$ del IVI total (Tabla 1).

La biofumigación con $2,5 \mathrm{~kg} \cdot \mathrm{m}^{-2}$ registró la menor cantidad de individuos de arvenses dicotiledóneas $(48,08 \%$ menos que el control) y se diferenció significativamente del control y de las otras dosis de $B$. juncea $(p \leq 0,021)$. Los tratamientos con 5 y $15 \mathrm{~kg} \cdot \mathrm{m}^{-2}$ no se diferenciaron significativamente del control. La biofumigación con $10 \mathrm{~kg} \cdot \mathrm{m}^{-2}$ registró la mayor cantidad de arvenses dicotiledóneas y se diferenció significativamente del resto $\left(p \leq 1,08 \times 10^{-3}\right.$ ) (Figura 1C).

\section{Cobertura total de arvenses}

Sólo el tratamiento con $2,5 \mathrm{~kg} \cdot \mathrm{m}^{-2}$ registró menor cobertura de arvenses que el control, pero la diferencia no fue significativa. La cobertura en el tratamiento biofumigante con $5 \mathrm{~kg} \cdot \mathrm{m}^{-2}$ fue estadísticamente similar a la del control. En las dosis de 10 y $15 \mathrm{~kg} \cdot \mathrm{m}^{-2}$ se observaron los valores más altos de cobertura de arvenses, que difirieron significativamente del control $(p$ $\leq 0,011$ ) (Figura 1-D).

\section{Peso seco total de arvenses}

Los tratamientos biofumigantes con 2,5 y $5 \mathrm{~kg} \cdot \mathrm{m}^{-2}$ no registraron diferencias significativas con el control para el parámetro peso seco de arvenses. Las dosis más altas de $B$. juncea (10 y $\left.15 \mathrm{~kg} \cdot \mathrm{m}^{-2}\right)$ mostraron los mayores pesos secos de arvenses y se diferenciaron significativamente del control $\left(p \leq 5,17 \times 10^{-3}\right)$ (Figura 1E).

\section{Cantidad de individuos por especie de arvense}

En la flora arvense del sustrato evaluado, siete especies registraron índices de valor de importancia superiores a 10 y representaron el $74,74 \%$ del IVI total: $D$. sanguinalis (pasto de cuaresma), Portulaca oleracea L. (verdolaga), Taraxacum officinalis G.H. Weber ex Wiggers (diente de león), Echinochloa spp., Trifolium repens L. (trébol blanco), Coronopus didymus (L.) Sm. (mastuerzo) y Picris echiodes L. (cerraja pegajosa). Estas especies se evaluaron individualmente mediante la comparación de la densidad por especie. Además, se observó la presencia de otras 10 especies arvenses, que registraron IVI inferiores a 10 (Tabla 1). 

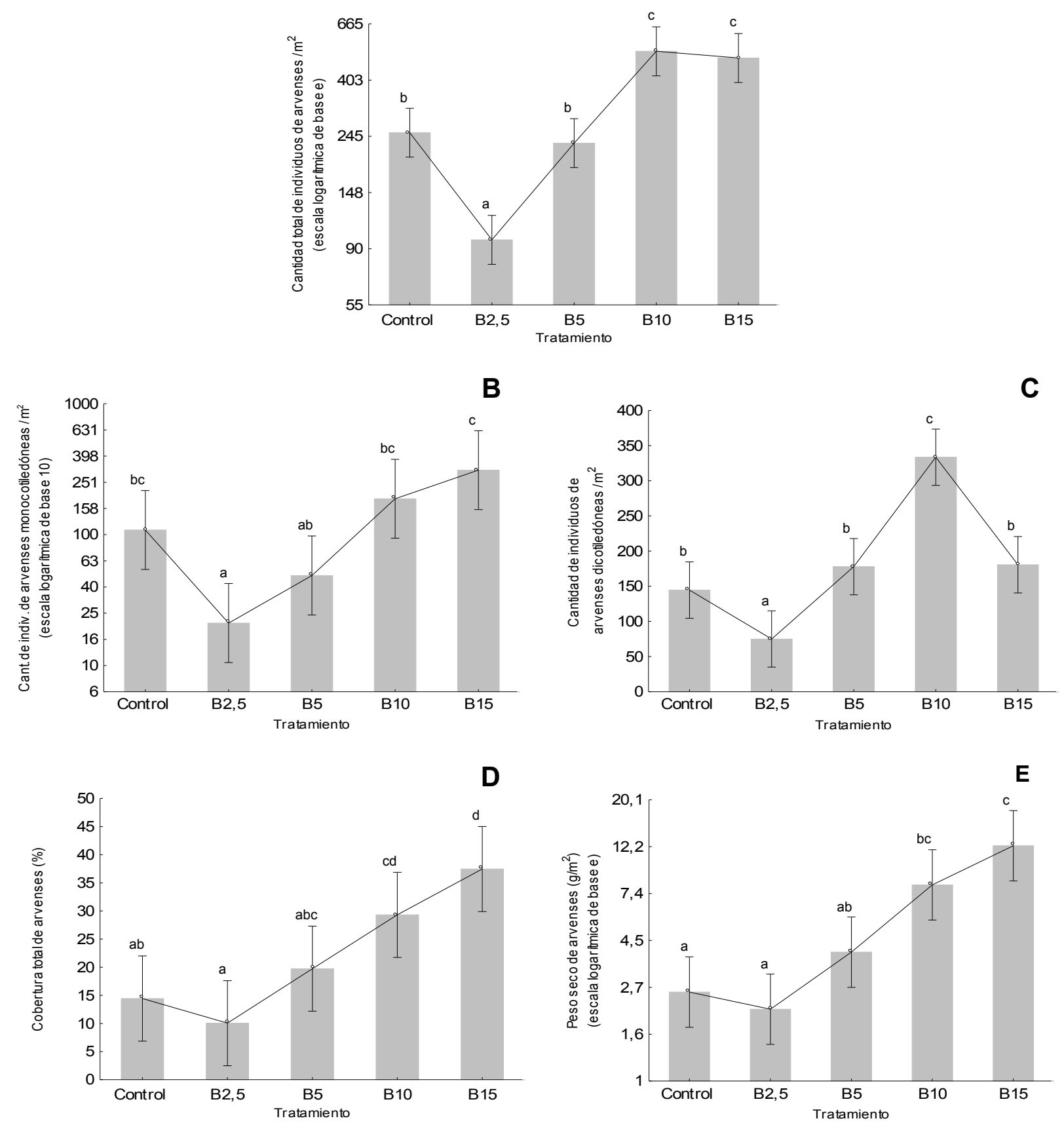

Figura 1. Efecto de la biofumigación con diferentes dosis de $\underline{B}$. juncea sobre la flora arvense, en función de los parámetros evaluados: cantidad total de individuos de arvenses $(A)$, cantidad de individuos de arvenses monocotiledóneas (B), cantidad de individuos de arvenses dicotiledóneas $(C)$, cobertura total de arvenses (D) y peso seco total de arvenses (E). Control: tratamiento sin biofumigación; B2,5, B5, B10 y B15: biofumigación con B. juncea en dosis de 2,5, 5, 10 y $15 \mathrm{~kg} \cdot \mathrm{m}^{-2}$, respectivamente. Las barras verticales indican intervalos de confianza del 95\%. Las letras distintas encima de las barras verticales indican diferencias significativas por la prueba de Tukey $(p<0,05)$.

\section{D. sanguinalis}

El pasto de cuaresma fue la especie predominante en la flora arvense, con un IVI relativo total (IVI/IVI total) de 37,96\%; representó el $80,57 \%$ del IVI de especies monocotiledóneas (Tabla 1). La biofumigación con 2,5 $\mathrm{kg} \cdot \mathrm{m}^{-2}$ registró la menor cantidad de individuos de esta especie arvense y se diferenció significativamente del control y de los tratamientos con 10 y $15 \mathrm{~kg} \cdot \mathrm{m}^{-2}(\mathrm{p} \leq$ 0,048 ). La dosis de $2,5 \mathrm{~kg} \cdot \mathrm{m}^{-2}$ provocó una reducción de la cantidad de individuos de pasto de cuaresma del $75,61 \%$. Las dosis más altas de B. juncea (10 y 15 $\mathrm{kg} \cdot \mathrm{m}^{-2}$ ) registraron las mayores cantidades de individuos de $D$. sanguinalis y no se diferenciaron 
significativamente del control, pero si del tratamiento con $5 \mathrm{~kg} \cdot \mathrm{m}^{-2}$ ( $\left.\mathrm{p} \leq 0,015\right)$ (Tabla 2).

Dado que $D$. sanguinalis predominó entre las malezas de hoja angosta, su presencia condicionó los resultados de la cantidad de individuos de arvenses monocotiledóneas.

\section{$P$. oleracea}

La verdolaga fue la especie con mayor IVI dentro de las dicotiledóneas y representó el $13,49 \%$ del IVI de especies de hoja ancha y el $7,14 \%$ del IVI total (Tabla 1). La biofumigación con $2,5 \mathrm{~kg} \cdot \mathrm{m}^{-2}$ registró la menor cantidad de individuos de $P$. oleracea y se diferenció significativamente del control $(p$ value $=0,021)$ y de las dosis de 10 y $15 \mathrm{~kg} \cdot \mathrm{m}^{-2}$ ( $\left.\mathrm{p} \leq 0,015\right)$. La dosis de 2,5 $\mathrm{kg} \cdot \mathrm{m}^{-2}$ provocó una reducción de la cantidad de individuos de verdolaga del $83,33 \%$. Los otros tratamientos no mostraron diferencias significativas con el control, ni entre sí (Tabla 2).

\section{T. officinalis}

El tratamiento con $2,5 \mathrm{~kg} \cdot \mathrm{m}^{-2}$ presentó la menor cantidad de individuos de $T$. officinalis y se diferenció significativamente del control y de los tratamientos biofumigantes ( $p \leq 0,042$ ). Los tratamientos con 5, 10 y $15 \mathrm{~kg} \cdot \mathrm{m}^{-2}$ no registraron diferencias significativas entre sí, ni con el control (Tabla 2).

\section{C. didymus}

La biofumigación con $2,5 \mathrm{~kg} \cdot \mathrm{m}^{-2}$ registró la menor cantidad de individuos de $C$. didymus y se diferenció significativamente de los tratamientos con 10 y 15 $\mathrm{kg} \cdot \mathrm{m}^{-2}(p \leq 5,54 \times 10-3)$ pero no del control. El tratamiento con $5 \mathrm{~kg} \cdot \mathrm{m}^{-2}$ no se diferenció del control ni de los tratamientos con 2,5 y $15 \mathrm{~kg} \cdot \mathrm{m}^{-2}$, pero sí de la dosis de $10 \mathrm{~kg} \cdot \mathrm{m}^{-2}\left(\mathrm{p}\right.$ value $\left.=2,97 \times 10^{-3}\right)$. El tratamiento con $10 \mathrm{~kg} \cdot \mathrm{m}^{-2}$ registró la mayor cantidad de individuos de $C$. didymus y se diferenció significativamente del control y de los demás tratamientos biofumigantes ( $p \leq$ $2,97 \times 10^{-3}$ ), salvo de la dosis de $15 \mathrm{~kg} \cdot \mathrm{m}^{-2}$ (Tabla 2).

Echinochloa spp., T. repens y $P$. echiodes No se registraron diferencias significativas entre tratamientos, en la cantidad de individuos de estas especies arvenses (Tabla 2).

\section{Resultados expresados en función de la dosis del biofumigante}

En el tratamiento con $2,5 \mathrm{~kg} \cdot \mathrm{m}^{-2}$ se registraron los valores más bajos de todos los parámetros evaluados, salvo la cantidad de individuos de $T$. repens y $P$. echiodes. Se hallaron diferencias significativas, con respecto al control, en la cantidad total de individuos de arvenses, monocotiledóneas y dicotiledóneas, en la cantidad de individuos de $D$. sanguinalis, $P$. oleracea y $T$. officinale, pero no en el peso seco total, en la cobertura total y en la cantidad de individuos de Echinochloa spp., T. repens, C. didymus y P. echioides. La dosis de $5 \mathrm{~kg} \cdot \mathrm{m}^{-2}$ no se diferenció significativamente del control en ninguno de los parámetros evaluados.

Los tratamientos biofumigantes con las dosis más altas de $B$. juncea $\left(10\right.$ y $15 \mathrm{~kg} . \mathrm{m}^{-2}$ ) registraron los mayores valores en todos los parámetros evaluados, con excepción de la cantidad de individuos de $T$. repens y $P$. echioides en el tratamiento con $15 \mathrm{~kg} \cdot \mathrm{m}^{-2}$. Se diferenciaron significativamente del control en la cantidad total de individuos de arvenses, la cobertura, el peso seco de la biomasa aérea y en la cantidad de individuos de C. didymus.

Tabla 1. Especies presentes en la flora arvense del sustrato evaluado. IVI: índice de valor de importancia; IVI relativo Total: IVIIIVI total; IVI relativo Monocot.: IVIIIVI monocotiledóneas; IVI relativo Dicot.: IVI/IVI dicotiledóneas. En negrita se resaltan las especies con IVI > 10.

\begin{tabular}{lcccc}
\hline & \multirow{2}{*}{ IVI } & \multicolumn{3}{c}{ IVI relativo (\%) } \\
& Total & Monocot & Dicot. \\
\hline Digitaria sanguinalis & 75,92 & 37,96 & 80,57 & - \\
Portulaca oleracea & 14,27 & 7,14 & - & 13,49 \\
Taraxacum officinalis & 13,88 & 6,94 & - & 13,12 \\
Echinochloa spp. & 12,71 & 6,35 & 13,49 & - \\
Trifolium repens & 11,47 & 5,74 & - & 10,85 \\
Coronopus didymus & 10,65 & 5,33 & - & 10,07 \\
Picris echiodes & 10,56 & 5,28 & - & 9,98 \\
Anoda cristata & 9,28 & 4,64 & - & 8,77 \\
Ligustrum spp. & 9,28 & 4,64 & - & 8,77 \\
Stachys arvensis & 9,18 & 4,59 & - & 8,68 \\
Galinsoga parviflora & 6,00 & 3,00 & - & 5,67 \\
Sorghum halepense & 2,80 & 1,40 & 2,97 & - \\
Oxalis articulata & 2.80 & 1,40 & - & 2,65 \\
Ammi visnaga & 2,80 & 1,40 & - & 2,65 \\
Galega officinalis & 2,80 & 1,40 & - & 2,65 \\
Stellaria media & 2,80 & 1,40 & - & 2,65 \\
Cyperus rotundus & 2,80 & 1,40 & 2,97 & - \\
Monocotiledóneas & 94,23 & 47,12 & 100,00 & - \\
Dicotiledóneas & 105,77 & 52,88 & - & 100,00 \\
\hline Total & 200,00 & 100,00 & - & - \\
\hline
\end{tabular}


Tabla 2. Densidad promedio (cantidad promedio de individuos por metro cuadrado) de las especies más importantes de la flora arvense del sustrato evaluado. D.s.: Digitaria sanguinalis; P.o.: Portulaca oleracea; T.o.: Taraxacum officinale; E. spp.: Echinochloa spp.; T.r.: Trifolium repens; C.d.: Coronopus didymus; P.e.: Picris echioides. Control: tratamiento sin biofumigación; B2,5, B5, B10 y B15: biofumigación con B. juncea en dosis de 2,5, 5, 10 y 15 kg.m ${ }^{-2}$, respectivamente. Los valores con letras distintas en una misma columna indican diferencias significativas por la prueba de Tukey $\left({ }^{1}\right)$ o Kruskal-Wallis $\left(^{2}\right)(p<0,05)$.

\begin{tabular}{|c|c|c|c|c|c|c|c|}
\hline & \multicolumn{7}{|c|}{ Densidad promedio (cantidad promedio de individuos $/ \mathrm{m}^{2}$ ) } \\
\hline & D.s. $^{1}$ & P.o. ${ }^{1}$ & T.o. ${ }^{1}$ & E. spp. ${ }^{2}$ & T.r. ${ }^{2}$ & C.d. ${ }^{1}$ & P.e. $^{1}$ \\
\hline Control & $113,98 \mathrm{bc}$ & $33,36 \mathrm{bc}$ & $22,24 b$ & $0,00 \mathrm{a}$ & $25,02 \mathrm{a}$ & 8,34 & $\overline{20,85 a}$ \\
\hline $\mathrm{B} 2,5$ & 27,80 a & 5,56 a & 8,34 a & $0,00 \mathrm{a}$ & 4,17 a & 4,17 & 31,97 a \\
\hline B5 & $45,87 a b$ & $23,63 a b$ & $55,60 \mathrm{~b}$ & 5,56 a & $8,34 a$ & $12,51 a b$ & 43,09 a \\
\hline B10 & $184,87 \mathrm{~cd}$ & $52,82 \mathrm{bc}$ & $47,26 \mathrm{~b}$ & $8,34 \mathrm{a}$ & 66,72 a & $88,96 \quad c$ & $55,60 a$ \\
\hline B15 & $282,17 \mathrm{~cd}$ & $34,75 \mathrm{bc}$ & $55,60 \mathrm{~b}$ & $16,68 \mathrm{a}$ & $1,39 a$ & $43,09 \mathrm{bc}$ & $34,75 \mathrm{a}$ \\
\hline
\end{tabular}

La dosis de $10 \mathrm{~kg} \cdot \mathrm{m}^{-2}$ también mostró diferencias significativas en la cantidad de arvenses dicotiledóneas.

\section{DISCUSIÓN}

La biofumigación con $B$. juncea influyó sobre la emergencia de las arvenses, de manera variable según la dosis aplicada.

Efecto de la biofumigación con $2,5 \mathrm{~kg} \cdot \mathrm{m}^{-2}$ de mostaza parda sobre las malezas

La dosis más baja de $B$. juncea $\left(2,5 \mathrm{~kg} \cdot \mathrm{m}^{-2}\right)$ fue eficaz para reducir la densidad de las arvenses; mostró una acción inhibidora sobre la emergencia de las malezas, ya que redujo el número de individuos de arvenses mono y dicotiledóneas, en particular de $D$. sanguinalis, $P$. oleracea y $T$. officinale. Sin embargo, no ralentizó significativamente el crecimiento de las plántulas emergidas, por lo que la cobertura y el peso seco totales fueron apenas inferiores a los del control. Probablemente, la menor densidad de plántulas en el tratamiento con $2,5 \mathrm{~kg} \cdot \mathrm{m}^{-2}$, con respecto al control, fue la causa del mayor crecimiento individual debido a una menor competencia por los recursos disponibles.

La reducción significativa de la cantidad de individuos de $P$. oleracea, también fue observada en estudios previos ejecutados por nuestro grupo de trabajo. En un ensayo realizado en condiciones de campo, donde se incorporó al suelo un cultivo de cobertura de mostaza parda en plena fructificación, en dosis de $1,68 \mathrm{~kg} \cdot \mathrm{m}^{-2}$ de biomasa aérea fresca, se observó reducción de la emergencia de P. oleracea y Anoda cristata (L.) Schltdl. (malva cimarrona) (Perniola et al., 2011). Si bien la dosis de mostaza parda fue inferior a los $2,5 \mathrm{~kg} \cdot \mathrm{m}^{-2}$ utilizados en el presente ensayo, tuvo un efecto similar sobre la emergencia de $P$. oleracea. En otro trabajo realizado in vitro, se evaluó la germinación de semillas de $A$. cristata, $P$. echiodes y $P$. oleracea sometidas a los gases biofumigantes de mostaza parda, dentro de recipientes de plástico de $900 \mathrm{ml}$ de capacidad; se utilizaron dos dosis de 10 y $40 \mathrm{~g}$ de material triturado de mostaza parda en el estadio de fin de fructificación. Se observó un marcado efecto inhibidor de la germinación de las tres especies arvenses evaluadas, con porcentajes de plántulas normales que en ninguno de los tratamientos superó el 5,5\% (Perniola et al., 2016). Estos resultados coinciden con los observados en $P$. oleracea, pero no en $P$. echiodes. En otro ensayo desarrollado por nuestro grupo de trabajo (Perniola et al., inédito, 2018), se aplicó una metodología similar a la descripta en la presente investigación, con incorporación de 2,26 kg.m ${ }^{-2}$ de biomasa aérea fresca de mostaza parda en el estadio de fin de fructificación y posterior riego y cobertura con polietileno negro durante 21 días; se midió la emergencia de $P$. oleracea a los 20 días de retirada la cobertura de polietileno y se observó una disminución del $83,4 \%$ en la cantidad de individuos y del $90,9 \%$ en el peso seco total, con respecto al control. La reducción del número de individuos de $P$. oleracea coincidió con la observada en el presente trabajo, que fue de $83,3 \%$ con una dosis de $2,5 \mathrm{~kg} \cdot \mathrm{m}^{-2}$. Los resultados obtenidos con la dosis de $2,5 \mathrm{~kg} \cdot \mathrm{m}^{-2}$, coincidieron parcialmente con los hallados por otros autores. Dado que la efectividad de la biofumigación depende de numerosos factores, como se mencionó en la introducción de este trabajo, en muchos casos resulta difícil establecer comparaciones con otras investigaciones. Norsworthy et al. (2007) determinaron visualmente una reducción de $D$. sanguinalis del $73 \%$ con respecto al control sin biofumigante, 17 días después de la incorporación de un cultivo de cobertura de $B$. juncea "F-E75". Este cultivo biofumigante, cuando alcanzó el estadio de mediados a fin de fructificación, se trituró e incorporó a razón de 617 g.m ${ }^{-2}$ de biomasa aérea seca, pero no se cubrió el suelo con polietileno. La dosis fue un $30 \%$ mayor que la dosis de $2,5 \mathrm{~kg} \cdot \mathrm{m}^{-2}$ (equivalente a 475 g.m ${ }^{-2}$ de biomasa aérea seca) utilizada en el presente ensayo; no obstante, el control de D. sanguinalis resultó similar. Wang et al. (2015) hallaron que la biofumigación con HSM de $B$. juncea "Pacific Gold", en dosis de 50 a 339 g/m² de biomasa seca, fue efectiva para reducir el porcentaje y el índice de emergencia de $D$. sanguinalis, con un incremento del efecto bioherbicida a medida que aumentó la dosis, dentro del rango antes señalado. En este caso, las dosis fueron del $10,5 \%$ al $71,4 \%$ de la dosis de 475 g. $\mathrm{m}^{-2}$ utilizada en el presente ensayo, pero se biofumigó con HSM y no con plantas frescas trituradas. Vaughn et al. (2006) también observaron efectos herbicidas de la biofumigación con $B$. juncea; hallaron inhibición completa de la emergencia de plántulas de Triticum aestivum L. y de la maleza Senna obtusifolia (L.) H. S. Irwin \& Barneby sembradas en macetas, cuando incorporaron a un sustrato 0,1 y $0,5 \%$ (peso/peso) de HSM de $B$. juncea, respectivamente. La dosis más alta 
aplicada por Vaughn et al. fue similar a la dosis de 2,5 $\mathrm{kg} \cdot \mathrm{m}^{-2}$ utilizada en el presente ensayo (equivalente a $0,52 \%$ [peso seco del biofumigante/peso del sustrato]), pero el tipo de material biofumigante fue distinto.

\section{Efecto de la biofumigación con $5 \mathrm{~kg} \cdot \mathrm{m}^{-2}$ de mostaza parda sobre las malezas}

El tratamiento biofumigante con $5 \mathrm{~kg} \cdot \mathrm{m}^{-2}$ de $B$. juncea mostró un comportamiento similar al control sin biofumigante. Debido a que el tratamiento control es per se una táctica de manejo de malezas conocida como cobertura plástica de suelo, acolchado, mulch o mulching (Anzalone et al., 2011; Kasirajan \& Ngouajio, 2012; Lament, 1993; Ngouajio \& Ernest, 2004), la biofumigación con $5 \mathrm{~kg} \cdot \mathrm{m}^{-2}$ también podría considerarse una estrategia de manejo de malezas. No obstante, su aplicación resultaría más laboriosa y costosa que la biofumigación con $2,5 \mathrm{~kg} \cdot \mathrm{m}^{-2}$, ya que requeriría duplicar la superficie destinada a obtener el biofumigante.

Otros investigadores observaron efectos sobre las malezas cuando biofumigaron con dosis cercanas a 5 $\mathrm{kg} \cdot \mathrm{m}^{-2}$. Wang et al. (2008) incorporaron al suelo un cultivo de cobertura de $B$. juncea $\left(823,4 \mathrm{~g}_{\mathrm{m}} \mathrm{m}^{-2}\right.$ de biomasa seca, estadio fenológico no especificado) previo a la siembra de Allium cepa L. y observaron reducción significativa de la densidad total de malezas y de la densidad de $P$. oleracea y Amaranthus retroflexus L., comparado con el control no precedido por $B$. juncea; cabe aclarar que el suelo no fue tapado con polietileno después de la incorporación de los cultivos de cobertura, aunque fue regado por aspersión para sellar la superficie edáfica y evitar pérdidas excesivas de gases biocidas volátiles. La cantidad de biofumigante incorporado fue similar a la dosis de 5 $\mathrm{Kg} \cdot \mathrm{m}^{-2}$ (equivalente a $950 \mathrm{~g} \cdot \mathrm{m}^{-2}$ de biomasa aérea seca) utilizada en el presente ensayo; no obstante, Wang et al. observaron acción herbicida, probablemente, debido a que los resultados del tratamiento biofumigante fueron comparados con un control sin cobertura plástica (a diferencia del presente ensayo). En un experimento realizado en invernadero, Krishnan et al. (1998) incorporaron $20 \mathrm{~g}$ de tallos y hojas frescos y triturados de $B$. juncea, en el estadio de principios de floración, en recipientes que contenían $454 \mathrm{~g}$ de suelo franco arcillo limoso $(4,4 \%$ [peso fresco del biofumigante/peso del sustrato]). En ese sustrato sembraron semillas de cinco especies de malezas y regaron periódicamente para mantenerlo en capacidad de campo; no utilizaron cobertura de polietileno sobre el sustrato. Observaron reducción de la emergencia y del peso fresco de Capsella bursa-pastoris (L.) Medikus, K. scoparia y $S$. viridis y solo reducción del peso fresco de $A$. retroflexus y Abutilon theophrasti Medik. Si bien la cantidad de biofumigante incorporado fue similar a la dosis de 5 $\mathrm{Kg} \cdot \mathrm{m}^{-2}$ empleada en el presente ensayo (equivalente a $5,5 \%$ [peso fresco de B. juncea/peso del sustrato]), Krishnan et al. observaron efectos supresores sobre las malezas. Esta disimilitud en los resultados podría deberse a diferencias en: el estadio fenológico de la mostaza parda, la cobertura del sustrato y las arvenses estudiadas. Norsworthy et al. (2005) encontraron reducción del $68 \%$ en la densidad de $D$. sanguinalis en cultivos de Vigna unguiculata (L.) Walp precedidos por abonos verdes mixtos de $B$. juncea y $S$. alba, incorporados a principios de fructificación en dosis de
702 g.m $\mathrm{m}^{-2}$ de biomasa aérea seca, sin cobertura de polietileno. Esta dosis fue $26,1 \%$ menor que la dosis de $5 \mathrm{~kg} \cdot \mathrm{m}^{-2}$ (equivalente a $950 \mathrm{~g} \cdot \mathrm{m}^{-2}$ de biomasa aérea seca) utilizada en el presente trabajo; sin embargo, la reducción de la densidad de $D$. sanguinalis fue similar a la observada con aplicación de $2,5 \mathrm{~kg} \cdot \mathrm{m}^{-2}$ de material fresco de $B$. juncea (equivalente a $475 \mathrm{~g} \cdot \mathrm{m}^{-2}$ de biomasa aérea seca). Este comportamiento diferente podría deberse a una composición distinta de los isotiocianatos producidos por el abono verde mixto y/o a la falta de cobertura del suelo que habría facilitado el escape de una parte de los gases biofumigantes, por lo que la dosis real habría sido menor $y$, probablemente, comparable con nuestra dosis de $2,5 \mathrm{~kg} \cdot \mathrm{m}^{-2}$.

\section{Efecto de la biofumigación con 10 y $15 \mathrm{~kg} \cdot \mathrm{m}^{-2}$ de mostaza parda sobre las malezas}

Los tratamientos biofumigantes con las dosis más altas de $B$. juncea $\left(10\right.$ y $15 \mathrm{~kg} \cdot \mathrm{m}^{-2}$ ) incrementaron el número de plántulas, la cobertura y el peso seco de las malezas, lo que sustentaría la hipótesis de una acción estimulante de esas dosis de $B$. juncea sobre la germinación y/o emergencia de las arvenses. Al respecto, Saini (2009) concluyó que el AITC proveniente de $B$. juncea, en altas concentraciones, podría actuar como promotor del crecimiento, debido a su similitud estructural con el etileno, conocida fitohormona que promueve la germinación y rompe la dormición de las semillas, de manera dependiente de la dosis y la especie vegetal. Saini ensayó la germinación de semillas de Linum usitatissimum L. y Solanum lycopersicum L. con diferentes concentraciones de AITC (0 a $1 \mathrm{mM}$ ) y observó estimulación de la germinación, de ambas especies, con concentraciones superiores a $0,1 \mathrm{mM}$; en cambio, a bajas concentraciones de AITC $(0,01$ y $0,05 \mathrm{mM})$ hubo depresión de la germinación. Si bien en nuestro ensayo no realizamos mediciones de concentración de isotiocianatos, los resultados mostraron una relación entre emergencia de malezas y dosis de $B$. juncea similar a la observada por Saini en semillas de $L$. usitatissimum y S. lycopersicum.

En los tratamientos con 10 y $15 \mathrm{~kg} \cdot \mathrm{m}^{-2}, D$. sanguinalis, la especie predominante de la flora arvense, registró una germinación $62,2 \%$ y $147,6 \%$ superior al control, respectivamente. Estas diferencias no fueron estadísticamente significativas; no obstante, fue evidente que existió un notable incremento del número de plantas, que podría explicarse por la estimulación de la germinación y/o la ruptura de la dormición de las semillas de $D$. sanguinalis. Al respecto, Teasdale \& Taylorson (1986) ensayaron el efecto del metil isotiocianato sobre semillas de $D$. sanguinalis y observaron que las concentraciones de 0,1 a $1 \mathrm{mM}$ estimularon la germinación de las semillas en dormición.

Otros autores también observaron efectos promotores de la germinación producidos por isotiocianatos, que podrían relacionarse con el comportamiento de los tratamientos de 10 y $15 \mathrm{~kg} \cdot \mathrm{m}^{-2}$. Auger et al. (2012) observaron que los exudados de 2-feniletil isotiocianato presentes en la rizósfera de Brassica napus L. estimularon la germinación de la maleza parásita Phelipanche ramosa L. Pomel. 


\section{CONCLUSIONES}

La biofumigación con plantas frescas de $B$. juncea, en el estadio de fin de fructificación, tiene un efecto variable sobre la emergencia de las malezas, en función de la dosis del biofumigante.

La dosis de $2,5 \mathrm{~kg} \cdot \mathrm{m}^{-2}$ de $B$. juncea es la única que resulta eficaz para reducir el número total de individuos de arvenses mono y dicotiledóneas, en particular de $D$. sanguinalis, $P$. oleracea y $T$. officinale, pero no produce una merma significativa de la biomasa aérea y la cobertura total.

La biofumigación con $5 \mathrm{~kg} \cdot \mathrm{m}^{-2}$ de $B$. juncea tiene una acción sobre las malezas, similar a la que genera la cobertura del suelo con polietileno negro.

Las dosis de 10 y $15 \mathrm{~kg} \cdot \mathrm{m}^{-2}$ de $B$. juncea incrementan la densidad, cobertura y biomasa de las arvenses.

Los resultados de este ensayo confirman los efectos favorables de la técnica de biofumigación con $2,5 \mathrm{~kg} \cdot \mathrm{m}^{-2}$ de $B$. juncea, para disminuir la densidad de las malezas $D$. sanguinalis, $P$. oleracea y $T$. officinale. Esta táctica representa una herramienta alternativa para el manejo integrado de malezas en estadios iniciales de cultivos hortícolas.

\section{BIBLIOGRAFÍA}

Altieri, M.A. \& C.I. Nicholls. 1999. Biodiversity, ecosystem function, and insect pest management in agricultural systems. In: Biodiversity in Agroecosystems. Collins W.W. \& Qualset C.O. (editors). Lewis Publisher, Boca Raton. pp. 69-84.

Anderson, D., J.B. Masiunas, S. Bossu \& M. Kushad. 2008. Mustards as biofumigants: current status and future prospects. North Central Weed Sci. Soc. Abstr. 63: 172. [CD-ROM computer file]. North Central Weed Sci. Soc., Champaign, Illinois, USA.

Anzalone, A., H. Ramírez-Guerrero, J. Lugo, A. Cirujeda, C. Zaragoza \& J. Aibar. 2011. Evaluación de cubiertas de suelo para el control de malezas en la producción integrada de tomate. Rev. Fac. Agron. (LUZ) 28: 71-90.

Auger, B., J.B. Pouvreau, K. Pouponneau, K. Yoneyama, G. Montiel, B. Le Bizec, K. Yoneyama, P. Delavault, R. Delourme \& P. Simier. 2012. Germination Stimulants of Phelipanche ramosa in the Rhizosphere of Brassica napus Are Derived from the Glucosinolate Pathway. Molecular Plant-Microbe Interactions 25 (7): 993-1004.

Bangarwa, S.K. \& J.K. Norsworthy. 2014. Brassicaceae Cover-Crop Effects on Weed Management in Plasticulture Tomato. Journal of Crop Improvement 28: 145-158.

Biaggi, C., F. Mondzak \& A. Valeiro. 2011. Problemas ambientales, oportunidades de desarrollo: la experiencia del Proyecto INTA Prozono. Ediciones INTA. Tucumán. 118 pp.

Björkman, T., C. Lowry, J.W. Shail, D.C. Brainard, D.S. Anderson \& J.B. Masiunas. 2015. Mustard Cover Crops for Biomass Production and Weed Suppression in the Great Lakes Region. Agronomy Journal 107(4): 1235-1249.

Blanco, Y. \& Á. Leyva. 2007. Las arvenses en el agroecosistema y sus beneficios agroecológicos como hospederas de enemigos naturales. Cultivos Tropicales 28(2): 21-28.

Boydston, R.A., T. Anderson \& S.F. Vaughn. 2008. Mustard (Sinapis alba) seed meal suppresses weeds in container-grown ornamentals. HortScience 43(3): 800803.

Boydston, R.A., M.J. Morra, V. Borek, L. Clayton \& S.F. Vaughn. 2011. Onion and weed response to mustard (Sinapis alba) seed meal. Weed Science 59: 546-552.

Brown, P.D. \& M.J. Morra. 1995. GlucosinolateContaining Plant Tissues as Bioherbicides. J. Agric. Food Chem. 43: 3070-3074.

Charron, C.S. \& C.E. Sams. 1999. Inhibition of Pythium ultimum and Rhizoctonia solani by shredded leaves of Brassica species. J. Amer. Soc. Hort. Sci. 124(5): 462467.

Earlywine, D.T., R.J. Smeda, T.C. Teuton, C.E. Sams \& X. Xiong. 2010. Evaluation of oriental mustard (Brassica juncea) seed meal for weed suppression in turf. Weed Technology 24(4): 440-445.

Ferrari, D.M., O.R. Pozzolo \& H.J. Ferrari. 2009. Desarrollo de un software para la estimación de la cobertura vegetal. INTA PROCISUR Informa. 5 pp.

Geary, B., C. Ransom, B. Brown, D. Atkinson \& S. Hafez. 2008. Weeds, disease, and nematode management in onions with biofumigants and metham sodium. HortTechnology 18: 569-574.

Gimsing, A.L. \& J.A. Kirkegaard. 2009. Glucosinolates and biofumigation: fate of glucosinolates and their hydrolysis products in soil. Phytochem Rev. 8: 299-310.

Handiseni, M., J. Brown, R. Zemetra \& M. Mazzola. 2011. Herbicidal activity of Brassicaceae seed meal on wild oat (Avena fatua), italian ryegrass (Lolium multiflorum), redroot pigweed (Amaranthus retroflexus), and prickly lettuce (Lactuca serriola). Weed Technology 25(1): 127-134.

Haramoto, E.R. \& E.R. Gallandt. 2005. Brassica cover cropping: I. Effects on weed and crop establishment. Weed Science 53: 695-701.

Hoagland, L., L. Carpenter-Boggs, J.P. Reganold \& M. Mazzola. 2008. Role of native soil biology in Brassicaceous seed meal-induced weed suppression. Soil Biology and Biochemistry 40: 1689-1697.

Iriarte, L.E., M.C. Sosa \& G.E. Reybet. 2011. Efecto de la biofumigación con repollo sobre el control de Fusarium oxysporum en suelo. RIA 37(3): 231-237.

Disponible

http://www.redalyc.org/articulo.oa?id=86421245007.

Último acceso: marzo de 2018.

Kasirajan, S. \& M. Ngouajio. 2012. Polyethylene and biodegradable mulches for agricultural applications: a review. Agron. Sustain. Dev. 32: 501-529.

Kirkegaard, J.A., P.A. Gardner, J.M. Desmarchelier \& J.F. Angus. 1993. Biofumigation using Brassica species to control pests and diseases in horticulture and agriculture. Proceedings of $9^{\text {th }}$ Australian Research Assembly on Brassicas. Wagga Wagga, Australia. pp. 77-82.

Kirkegaard, J.A., P.T.W. Wong \& J.M. Desmarchelier. 1996. In vitro supression of fungal root pathogens of cereals by Brassica tissues. Plant Pathol. 45: 593-603. 
Kjaer, A. 1976. Glucosinolates in cruciferae. En: The Biology and Chemistry of the Cruciferae. Academic Press, London. pp. 207-219.

Krishnan, G., D.L. Holshouser \& S.J. Nissen. 1998. Weed Control in Soybean (Glycine max) with Green Manure Crops. Weed Technology 12: 97-102.

Labrada, R. 1996. Manejo de malezas en hortalizas. En: Manejo de malezas para países en desarrollo. FAO, Roma. pp. 298-308.

Labrador Moreno, J. \& M. Altieri. 1994. Manejo y diseño de sistemas agrícolas sustentables. Hoja Divulgadora 6-7/94. Ministerio de agricultura, pesca y alimentación, Madrid, España. 52 pp.

Lafi, J.G., A.M. Tarquini, M. Sanz Pérez \& M.C. Puglia. 2017. Susceptibilidad in vitro de Fusarium spp, patógenas en tomate, a biofumigación con Brassicáceas. Procede de $4^{\circ}$ Congreso Argentino de Fitopatología. Mendoza, Argentina. pp. 363.

Lament, W.J. 1993. Plastic Mulches for the Production of Vegetable Crops. HortTechnology 3(1): 35-39.

Lefebvre, M., M. Leblanc \& A.K. Watson. 2013. Germination et survie de semences de mauvaises herbes à la biofumigation. Procede de $18 \mathrm{e}$ édition Les Journées horticoles. Saint-Rémi, Canada.

Mayton, H.S., C. Olivier, S.F. Vaughn \& R. Loria. 1996. Correlation of fungicidal activity of Brassica species with allyl-isotiocyanate production in macerated leaf tissue. Phytopathology 86: 267-271.

MBTOC (Methyl Bromide Technical Options Committee). 1994. Report of the Methyl Bromide Technical Options Committee. Montreal Protocol on substances that deplete the ozone layer. UNEP, Kenya. $304 \mathrm{pp}$.

Menalled, F.D. 2010. Consideraciones ecológicas para el desarrollo de programas de manejo integrado de malezas. Agroecología 5: 73-78.

Mitidieri, M., V. Brambilla, V. Saliva, E. Piris, M. Piris, R. Celié, C. Pereyra, K. Del Pardo, E. Chaves \& J. González. 2009. Efecto de distintas secuencias de tratamientos de biofumigación sobre parámetros fisicoquímicos y biológicos del suelo, el rendimiento y la salinidad de cultivos de tomate y lechuga bajo cubierta. Horticultura Argentina 28(67): 5-17.

Molina-Vargas, L.F. \& J.U. Bentura-Castellanos. 2009. Efecto inhibitorio in vitro de cinco isotiocianatos sobre Rhizoctonia solani Kühn AG-3. Revista de Investigación Agraria y Ambiental: 37-40.

Mostacedo, B. \& T.S. Fredericksen. 2000. Manual de métodos básicos de muestreo y análisis en ecología vegetal. Proyecto de manejo forestal sostenible (BOLFOR). Ed. El País. Santa Cruz, Bolivia. 87 pp.

Ngouajio, M. \& J. Ernest. 2004. Light transmission through colored polyethylene mulches affects weed populations. HortScience 39(6): 1302-1304.

Norsworthy, J.K., L. Brandenberger, N.R. Burgos \& M. Riley. 2005. Weed suppression in Vigna unguiculata with a spring-seeded brassicaceae green manure. Crop Protection 24(5): 441-447.

Norsworthy, J.K., M.S. Malik, P. Jha \& M.B. Riley. 2007. Suppression of Digitaria sanguinalis and Amaranthus palmeri using autumn-sown glucosinolateproducing cover crops in organically grown bell pepper. Weed Research 47: 425-432.

Páez, C. 2001. Algunos aspectos fitosociológicos y anatómicos de las principales gramíneas malezas en campos de arroz en el sistema de riego río Guárico. Tesis de maestría. Facultad de Agronomía, Universidad Central de Venezuela, Maracay, Venezuela. 162 pp.

Pattison, T., T. Martin, S. Akiew, C. Versteeg \& J. Kirkegaard. 2003. Can Brassicas be used to manage root-knot nematode in tropical vegetal production? Australasian Nematology Newsletter 14(2): 16-19.

Perniola, O.S., S.E. Chorzempa, S. Staltari, H. Rodriguez \& M. del C. Molina. 2011. Incidencia de la biofumigación sobre el control de malezas. Procede de VIII Simposio Nacional de Biotecnología REDBIO. C.A.B.A., Argentina. Sección 3. 54.

Perniola, O.S., S. Staltari, S.E. Chorzempa \& M. del C. Molina. 2012. Biofumigación con Brassicáceas: actividad supresora sobre Fusarium graminearum. Rev. Fac. Agron. 111(1): 48-53. Disponible en: http://www.agro.unlp.edu.ar/revista/index.php/revagro/ar ticle/view/71/46. Último acceso: marzo de 2018.

Perniola, O.S., S. Staltari, S.E. Chorzempa, M.M. Astiz Gassó \& M. del C. Molina. 2014. Control biológico de Fusarium graminearum: utilización de Trichoderma spp. y biofumigación con parte aérea de Brassica juncea. Rev Fac Cienc Agrar. 46(2): 45-56.

Perniola, O.S., S.E. Chorzempa, S. Staltari \& M. del C. Molina. 2016. Biofumigación in vitro con Brassica juncea y Sinapis alba. Inhibición de la germinación y del crecimiento de plántulas de malezas. Rev. Fac. Agron. 115(1): 91-98.

Perniola, O.S., S.E. Chorzempa \& M. del C. Molina. 2018. Biofumigación con Brassica juncea: acción sobre la emergencia y el crecimiento de plántulas de Portulaca oleracea L. Manuscrito inédito.

Pizano, M. 2014. Eliminación del bromuro de metilo en países en vías de desarrollo. Una historia de éxito y sus retos. Programa de las Naciones Unidas para el Medio Ambiente. París. 60 pp.

Price, A.J., C.S. Charron, A.M. Saxton \& C.E. Sams. 2005. Allyl Isothiocyanate and Carbon Dioxide Produced during Degradation of Brassica juncea Tissue in Different Soil Conditions. HortScience 40(6): 17341739.

Pung, H., S. Cross \& D. Patten. 2008. The use of biofumigant green manure crops for soil-borne disease management in Tasmania. Proceedings of Third

International Biofumigation Symposium. Canberra, Australia. pp. 26.

Saini, A.R.K. 2009. Aspects of Brassica juncea meal toxicity: allyl isothiocyanate release and bioassay. M. Sc. Tesis. Department of Food and Bioproduct Sciences, University of Saskatchewan, Saskatoon, Canada. $117 \mathrm{pp}$

Teasdale, J.R. \& R.B. Taylorson. 1986. Weed Seed Response to Methyl Isothiocyanate and Metham. Weed Science 34(4): 520-524.

Vaughn, S.F., D.E. Palmquist, S.M. Duval \& M.A. Berhow. 2006. Herbicidal activity of glucosinolatecontaining seedmeals. Weed Science 54: 743-748.

Wang, G., M. Ngouajio \& D.D. Warncke. 2008. Nutrient Cycling, Weed Suppression, and Onion Yield Following Brassica and Sorghum Sudangrass Cover Crops. HortTechnology 18(1): 68-74.

Wang, X., M. Gu, G. Niu \& P.A. Baumann. 2015. Herbicidal activity of mustard seed meal (Sinapis alba "IdaGold" and Brassica juncea "Pacific Gold") on weed 
Revista de la Facultad de Agronomía, La Plata (2019) Vol 118 (1): 25-35

emergence. Industrial Crops and Products 77: 10041013.

Webber, C.L., J.W. Shrefler \& L.P. Brandenberger. 2012. Organic Weed Control. In: Herbicides Environmental Impact Studies and Management Approaches. Alvarez-Fernandez R. (Ed.). Publisher: InTech. Rijeka, Croatia. pp. 185 -198. Disponible en: https://www.intechopen.com/books/herbicidesenvironmental-impact-studies-and-managementapproaches/organic-weed-control. Último acceso: marzo de 2018.

Yu, J. \& D.W. Morishita. 2014. Response of seven weed species to corn gluten meal and white mustard (Sinapis alba) seed meal rates. Weed Technology 28(1): 259-265. 